\title{
Research on Design of Offline Luggage Stores Based on User Experience
}

\author{
Jianyao Shu ${ }^{1}$, Xitong $\mathrm{Hu}^{2}$, Weiguo Zhang ${ }^{3}$, and Yun Mei* \\ ${ }^{1}$ Industrial design, University of Science and Technology Liaoning, Anshan City, Liaoning Province, 114051, China
}

\begin{abstract}
In today's fashion field, in addition to clothing and footwear, luggage also has most of the consumer market. Bags and luggage products are different from consumer goods such as clothing and accessories. The luggage market has greater potential and expansion capacity. With the advent of 2020, major progress has been made in building a well-off society in an all-round way, the level of the national economy has continued to improve, and the quality of life of our residents has become better and better. Consumers purchasing items not only consider quality and function, but also put forward requirements on quality and personalization. Compared with the traditional offline luggage stores, experiential offline stores pay more attention to consumers' purchase process, optimize the purchase process, and satisfy the spiritual consumption experience. Today, many brands have begun to pay attention to the experience design of offline physical stores, but it is not enough, especially for luggage stores. In the era of the explosion of the experience economy, exploring the user experience and integrating experience design into the physical stores of luggage and bags has become a research hotspot in the new era.
\end{abstract}

\section{User experience and experience design}

\subsection{User experience}

In the mid-1990s, the concept of user experience was first proposed by Donald Norman. He believes that a good product can enhance the feeling of the mind and thought at the same time, so that users have a pleasant feeling to appreciate, use and own it. User experience is a purely subjective experience established by users in the process of using the product. Design should be based on human feelings and people-oriented, but now many designs do the opposite, reflecting the "material-oriented" feature, so that users around the product, instead of using things. For example, when a WeChat account is logged in on a computer, you need to click on the mobile client to confirm. It often happens that you forgot to bring your mobile phone and you need to $\log$ in to WeChat. At this time, a lot of troubles will occur. After many process operations, it still cannot be smooth Log in to the WeChat computer client. The original intention of using a mobile phone to log in to the computer is to protect user privacy and ensure that I am logged in, but it does bring an unpleasant user experience in the actual operation process.

\subsection{Experience design}

Experience design is the integration of consumer participation in design, with service as the "stage", product as the "props", and environment as the "scenery" in an effort to make consumers feel a good experience in the course of business activities. Due to the booming national economy today, residents have changed from the need for material life to the pursuit of spiritual level. From the Maslow demand level(Fig.1), we can see that human needs are divided into three stages, from primary to advanced. The needs of this stage will further pursue the needs of the next stage and finally realize self. The experiential business space pays more attention to the consumer experience process and meets the demand for respect in Maslow's demand theory, which can further stimulate consumption and drive economic growth.

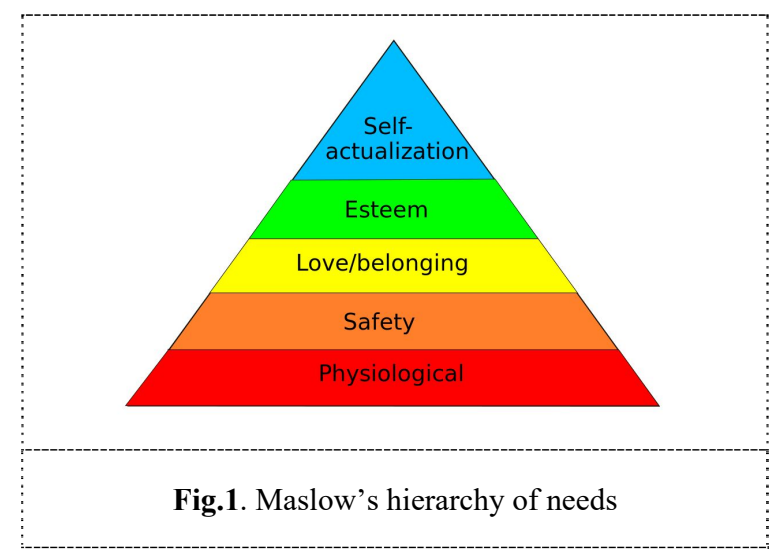




\section{Improve the significance of user experience in offline stores}

\subsection{Luggage consumer market}

In recent years, the Chinese luggage market has shown an upward trend. Leather goods account for $30 \%$ of the retail industry, especially women's luggage. Women usually play an important role in the consumer market. Cases and bags belong to the category of leather products. They share characteristics with clothing, trend elements change too quickly, design products iterate and update quickly, and consumers pursue fashionable and innovative products. China has a population of 1.4 billion and a per capita GDP of more than 10,000 US dollars, including about 400 million middle-income groups. Consumer demand for high-quality goods and services is growing. In recent years, the market size of China's luggage industry has gradually increased(Fig.2). Therefore, China's luggage market has huge consumption potential. Improving the user experience of offline stores is conducive to stimulating consumers' desire to purchase, completing purchase behaviors, and increasing recognition of the brand, and narrowed the relationship between users and brands.

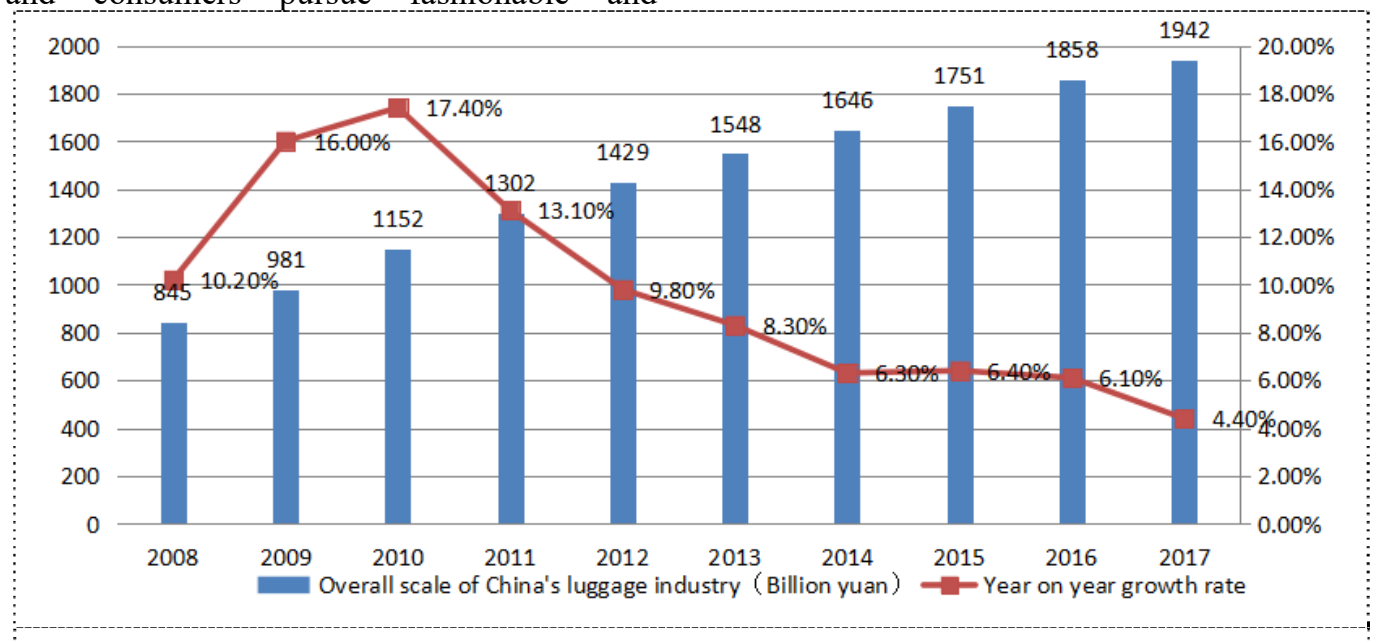

Fig.2. Market scale trend of China's luggage industry from 2008 to 2018 Image source: Euromonitor Research Institute for Advanced Industries

\subsection{Traditional luggage and offline stores}

As the market with the largest consumer potential, the market is currently divided into four parts(Fig.3): luxury brands, mid-end and high-end brands sold in shopping malls; low-end brands flood the wholesale luggage market. Traditional offline luggage stores do not involve product development, design, and production processes. They mainly buy goods in large quantities and then sell them to consumers in small quantities to earn the difference. Usually offline physical stores are located in stores with a large number of people, and through a series of discounts, buys and other promotions to attract consumers to shop. For offline sales stores in the traditional mode of luggage, shopping guides play an important role in the process of shopping for consumers. When consumers pick up a bag and test back, the shopping guide will interact with consumers. Have a greater impact on whether there is a purchase. For example, "Watson's" offline physical stores, when customers enter the door, they will have a welcoming shopping guide smile, and ask your purchase intentions, while continuing to promote certain products. For shopping guides, this may increase product sales, but for customers, it does cause confusion, which reduces the favorability of the brand, and even users will dislike the brand. There are many factors that affect customer consumption, as shown in Figure 4. Store design and service factors are important factors influencing purchases, so user experience is particularly important. The traditional sales model of shopping guides in stores is no longer in line with the current development trend. A series of store designs are needed to enhance the user experience, optimize the consumer experience, build the power of the brand, and build a quality brand. 


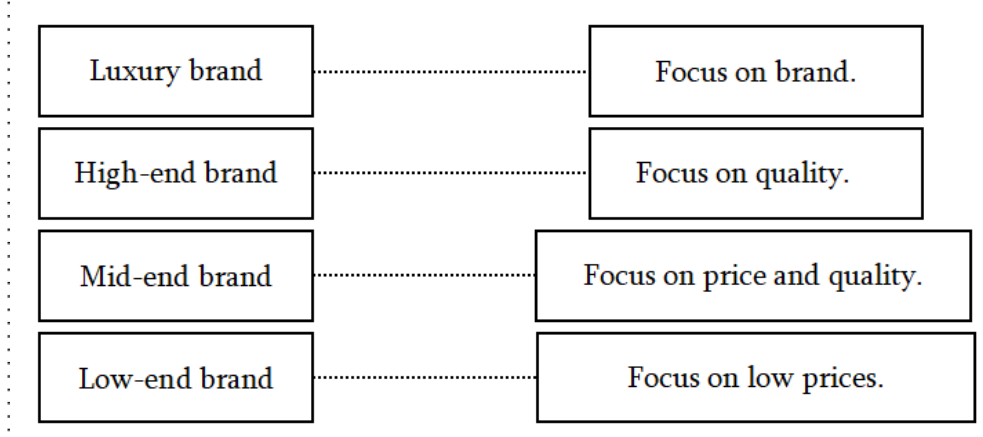

Fig.3. Chinese luggage industry brand classification

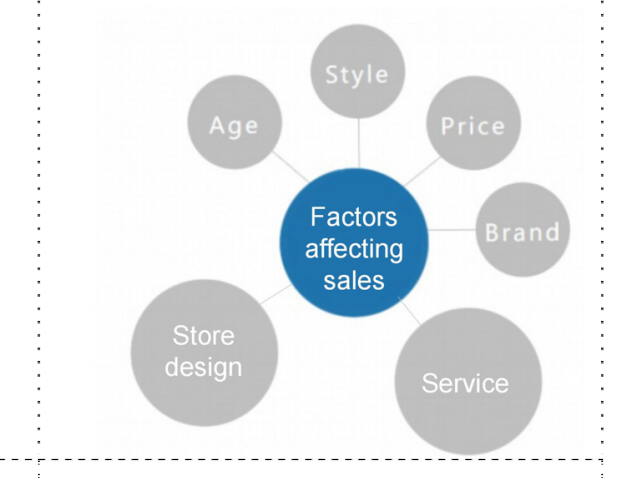

Fig.4. Factors affecting sales

\section{Research on the design and user experience of offline stores in luggage}

the elements of the store design and the shopping process, the moment of moving the customer is excavated to construct different experience level design methods, as shown in Figure 5:

Starting from the user experience level: the instinct level, the behavior level, and the reflection level, combining

\begin{tabular}{|c|c|}
\hline Experience level & Store design \\
\hline Instinct level & Store interior and exterior decoration design \\
\hline Behavioral level & Bag making process experience \\
\hline Reflection layer & Oriental traditional culture reappears \\
\hline
\end{tabular}

Fig.5. The relationship between store design and experience level

\subsection{Instinct-level offline luggage store design}

The instinct level is the user's sensory experience. The sensory experience refers to the visual and auditory feelings presented to the user, and is generally presented through elements such as color, shape, audio and video. Dietrams mentioned in the ten principles of design: Good design is aesthetic. In addition to meeting the basic functional needs of consumers, the product must have a certain aesthetic sense. Just as everyone likes beautiful girls, users also like the "high-value" design. The impact of sight and hearing on the user is much stronger than that of words. The first feeling of design comes from sight and hearing. When there is little communication between the user and the design, the judgment of the user depends only on the first impression. The "impression" at this time is not rational thinking, so the "face value" seems very important.

The decoration of the store is the first impression for consumers. If the decoration is unique, it has already attracted consumers' attention, so that customers can be attracted to visit the store. The style of store decoration can reflect the quality and taste of the brand. The user immediately generates an expected value for the brand based on the first impression of the store decoration. The quality of the decoration determines the user's rating of the brand. The style of the decoration affects the user's judgment of a brand's luggage style. For example, the store decoration styles of the two categories of women's handbags and sports bags are very different. Most of the luggage market in the consumer market is occupied by women's bags. Women are the main consumers, and they are usually emotional animals. They often consume impulsively when shopping. The design style of luggage stores for professional women should be simple, stylish, and decent. The neutral colors such as black and white and gray should be used to highlight the ability and charm of working women. On the contrary, the main purchase group of sports bags is men, and the store design style should be stylish and dynamic. The color of the interior display design is bright and visually striking, so that the user can experience the feeling of youth and vitality.

\subsection{Line-level offline luggage store design}

The behavior level is the interactive experience. The interactive experience process between the user and the product is also the process of defining whether the product is "good to use." In the design of the offline sales store of luggage, if the scene experience is very good, 
then the shopping experience of the user in the store is very pleasant. By creating an immersive offline experience store for luggage, consumers can experience the production process of luggage at the same time as shopping. In the design of display space, some designer's workbenches are used to display design sketches and semi-finished products, and the design process of a finished package is completely displayed in front of customers. Set up a public working area in the store to provide customers with an opportunity to experience making bags on site for free, so that customers have the experience of turning ideas into reality. Such a manual experience attracts consumers to actively participate, stimulates potential interest, and makes customers feel more in need of luggage products. With the development of technology, smart luggage has become a new hotspot in the current luggage market. Through the use of new media technologies in stores, consumers are attracted to wear smart luggage, which shortens the distance between products and users, and increases users' awareness of smart luggage favorable impression.

\subsection{Rethinking offline luggage store design}

Compared to the first two levels, the reflection level is a more advanced level, that is, the emotional experience. Because it is a user's psychological experience, it reflects "psychological recognition." Let users feel the value and temperature of the product and brand, users can realize the identity, express their inner emotions through the product, and finally have the inner recognition and acceptance.

The design of offline stores should incorporate humanistic elements or traditional culture. When the user first comes into contact with the product, they usually only understand the meaning of the product expression based on surface cognition, but ignore the most important spiritual level of feeling obtained through the reflection level. The design of offline stores in the luggage category should focus on conveying brand culture, gaining user recognition, and resonating with users. This enables users to produce emotional experiences that last longer than sensory or interactive experiences. For example, the Chinese "bampo" brand, which is an original Chinese brand, focuses on Chinese-style products. This brand 's bags pay attention to the quality of the leather and the design sense of the style. Adhering to the brand concept of "creating beauty and spreading beauty", it is committed to using modern aesthetics and skills to interpret oriental traditional culture, and to raise the inspiration of life into a fashionable art. Every woman can enjoy the beauty of Chinese original art. In addition to the need to showcase products in the offline luggage stores, it is also necessary to create a brand culture atmosphere so that customers understand the story behind each bag and establish an emotional connection between the brand and the customer.

\section{Conclusion}

When the offline physical store experience design can impress users, then a good relationship can be established between the brand and the user. Brands and users not only generate a sales relationship. Offline stores have improved the grade of their products through personalized and creative designs, meeting the users' requirements for high-quality products and fulfilling their spiritual needs. Users have a high degree of trust in the brand. When choosing similar products, they will choose a brand with a better experience. In the design of offline stores such as luggage, realizing the instinctual and behavioral level experience can naturally produce the emotional experience of the reflection level. The user experience is not a means, but a purpose. The offline physical store is a space. Designers should always maintain the human-oriented principle to make users have a more enjoyable experience in this space.

\section{References}

1. Xin Xiangyang. From user experience to experience design [J]. Packaging Engineering, 2019, 40 (08): 60-67. More references

2. $\mathrm{Hu}$ Fei, Jiang Mingyu. Research on Experience Design: Problem Situation, Disciplinary Logic and Theoretical Trends [J]. Packaging Engineering, 2018, 39 (20): 60-75.

3. Chu Liyang. Talking about the application of humanity in technical aesthetics in luggage design [J] .Research of Fine Arts Education, 2019 (21): 88-90.

4. Zhiwei. Research on the design of luggage based on traditional culture [D] .Northern Polytechnical University, 2019. 